\title{
Application of Intelligent Lighting System in Sheep Farm Based on CC2530-WiFi
}

\author{
Yun Pan \\ School of XinYang College of Agriculture And Forestry, Xinyang 464000, China \\ py0272@126.com
}

\begin{abstract}
With the expansion of the scale of modern sheep farming, the monitoring and control of environmental parameters in sheep farms is an urgent problem to be solved. This paper uses the CC2530-WiFi-based system to design the intelligent lighting system for the sheep farm. The experiments results showed that the system is low cost, low power consumption and high reliability, and wireless sensor network can be used as an effective solution for intelligent sheep farm construction environment.
\end{abstract}

Keywords: Intelligent sheep farm, CC2530-WiFi, Lighting system

\section{Introduction}

In recent years, with the development of large-scale agriculture and modernization, intelligent monitoring network is more and more used in storage, cultivation ,breeding, industrial control and other areas, the establishment of an effective monitoring network has become a very important part of the modernization of agriculture technology. Traditional monitoring system uses wired monitoring equipment, wiring difficulties, in sheep breeding environment is difficult to achieve effective maintenance. With the rapid development of sensor technology and based on WiFi wireless network, the combination of the two wireless monitoring system to solve the sheep in the monitoring system construction line problems, no subject to environmental constraints, easy to expand, easy maintenance ${ }^{[1]}$.

In the sheep feeding process, according to the experiment of fattening lambs in 10 degrees and 32 degrees at two temperatures, daily with 4, 8,12, 16, 20, 24 hours of light. Where 24 hours of light for continuous, The rest were illuminated twice a day, the result is 12 hours or more weight gain are significantly reduced, the results are 12 hours up and down weight gain are significantly reduced. Therefore, at a temperature of 15 degrees Celsius, light 12 hours a day, the fastest lamb daily gain of 248 grams, Light 8,16 hours, weight gain rates were 59\% and $50 \%{ }^{[2]}$, the experiment proved that the light in the sheep fattening process plays an important role. In this paper, RF transceiver CC2530 chip as the core, to build a wireless lighting control ZigBee wireless sensor network.

\section{The system design}

System block diagram 


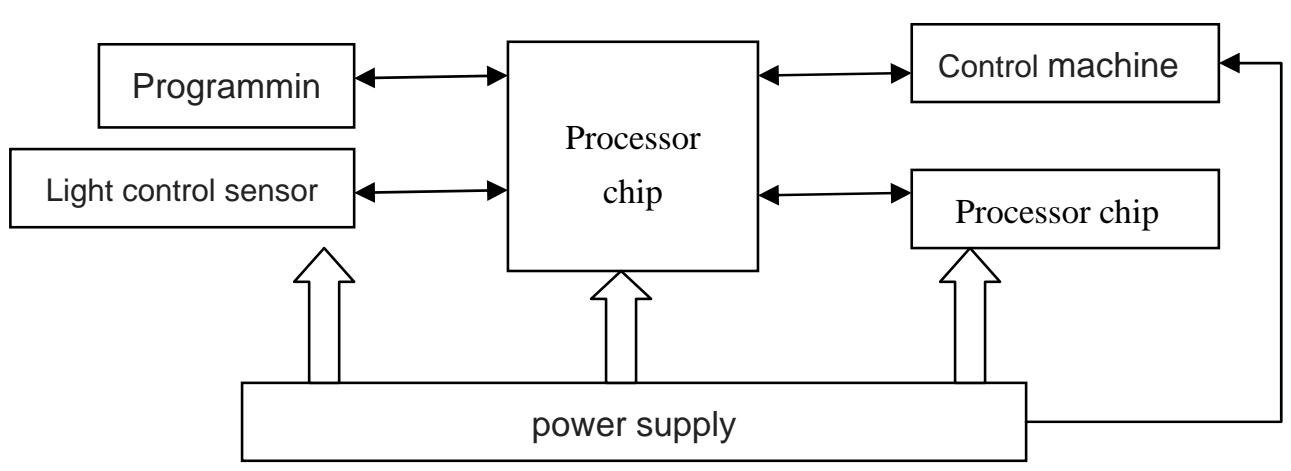

Figure 1 System overall block diagram

The system uses Zigbee developed sensor equipment, in the need to install the sensor inside the sheep factory, real-time collection of monitoring the parameters of the sheep's house, and then wirelessly transmitted to the network for collection and processing. System Design Block Diagram 1: System to single-chip terminal for the core, The system equipment mainly includes the microprocessor module, the wireless transmission module, the power module and so on. The light control sensor will receive the collected light to the microprocessor chip for processing, and then through the RF part of the information sent out to the control machine, the controller is responsible for collecting data and displayed on the screen, but also to the lower computer to convey the command.

System requirements

In the field of intelligent light control, the wireless acquisition system needs to meet the following aspects of the conditions:

1. Measure and collect data, select the wireless transmission communication.

2. In order to ensure the real-time data, the sensor node must send the lighting parameters accurately and on time.

3. In order to meet the long-term use of low power consumption, the system must have the ability to work continuously.

4. The system should have a higher reliability,if the individual nodes fail, do not affect the performance of the entire monitoring system.

5. Low cost, good expansion performance.

\section{Light monitoring system design}

Data Acquisition System

SCM selection $^{[3]}$

SCM choice, from the application system of SCM technical, practical, developable three aspects.

Technical: Mainly on the chip to choose. From the technical point of view to ensure that the SCM application system in a certain technical indicators to operate reliably

Practicality: to protect the SCM long-term, reliable operation, select the regular star manufacturers, so that the problems encountered in the latter part of the operation can be successfully resolved.

Development: SCM to have a reliable means of development, program development tools, simulation debugging tools, and so the tools are complete, with good development. 
ATMEGA16A-PU,which is manufactured by ATMEL,its main features: high-performance, low-power 8-bit AVR® microprocessors,advanced RISC architecture, 32 8-bit general purpose working registers, real-time counters with independent oscillators RT, Four-channel PWM.

Lighting sensor acquisition system

In this paper, the light sensor BH1750FVI module GY-30 (Figure 2), this non-integrated circuit can be based on the collected light intensity data to adjust the brightness of the lamp, and use its high resolution to monitor a wider range of light intensity changes.BH1750FVI module GY-30 is a digital light intensity sensor integrated circuit for a two-wire serial bus interface. This integrated circuit can be based on the collected light intensity data to adjust the LCD or keyboard backlight brightness

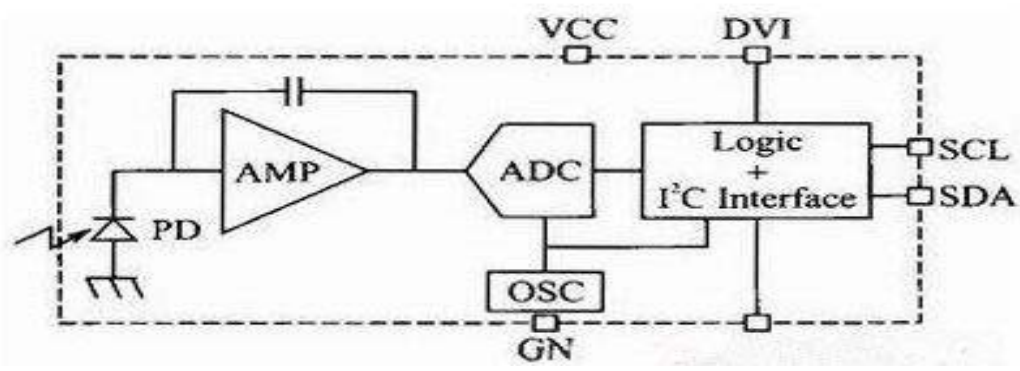

Figure 2 Block diagram of sensor BH1750FVI

System through the BH1750FVI module GY-30 collected light intensity, feedback to the ATMEGA16A-PU SCM, and the microcontroller connected to the LCD display real-time display of the collected light values. The data collected by the light sensor of the whole sheep field need to be transmitted to the SCM single-chip through the network. In the case of the small amount of data transmitted in the sheep farm, it is an ideal way to use the wireless data acquisition mode. This paper chooses the wireless based on Zigbee-WiFi transfer method.

Wireless transmission

Because of the Zigbee's low cost, low power consumption, short delay, high reliability, the system design of wireless acquisition system Zigbee technology. The Zigbee protocol cluster includes the physical layer, the MAC access control layer, the network layer, and the application layer. The next two layers follow the IEEE802.15.4 protocol, and the network layer above the level developed by the Zigbee Alliance (Figure 3).

\begin{tabular}{|c|c|}
\hline application layer & \\
\cline { 1 - 1 } API & \multirow{2}{*}{ Zigbee Alliance } \\
\cline { 1 - 1 } Security layer & \\
\cline { 1 - 1 } network layer & \\
\cline { 1 - 1 } MAC layer & \multirow{2}{*}{ IEEE802.15.4 } \\
\cline { 1 - 1 } PHY & \\
\hline
\end{tabular}

Figure 3 Zigbee protocol framework 
Zigbee network layer supports star topology and tree topology. Because tree topology has the advantage of star topology fault tolerance and can extend the distance of network, this paper chooses tree topology as network topology. The CC2530 is a low power, low voltage RF chip that conforms to the IEEE 802.15.4 protocol specification and is used in Zigbee networks to combine the performance of leading RF transceivers with system-programmable flash memory. Support for packet processing, data caching, data encryption, data validation, and free channel evaluation on hardware, it is also a low-frequency receiver.

The software design uses the Z-Stack protocol stack and the IAR integrated development environment that are compatible with the CC2530 chip. Z-Stack in the industry at the leading level, is still constantly improve and enhance, has been the world's many ZigBee application development manufacturers used to support a variety of hardware platforms. In addition, Z-Stack also supports a wealth of new features, such as wireless download, that is, through the ZigBee network, download nodes in the network upgrade software to complete the node software upgrades. In the global Zigbee system, the SCM controls the CC2530, performs bidirectional communication, sends control commands to it, and reads the status information of the CC2530 through the four-wire SPI bus. The ATMEGA16A-PU uses the interrupt to read and write the CC2530's first-in first-out register, the data transmitted in the wireless network, and sends the timing information to the CC2530 to identify the start of the data frame.

Lighting control system

In the whole sheep farm environment, due to the change of weather reasons, directly affect the light inside the sheep, according to the external light changes, the SCM received the light sensor data, and send drive instructions. In order to ensure that the most suitable amount of light to reach the sheep, this article uses a relay to control the installation of light inside the house. When the outside light to meet the needs of sheep, the sheep house light source closed. When the ambient light changes, such as cloudy, evening and other light is weak, the switch a few rows of light source, to reach the amount of light the amount of sheep. Lighting time control as early as 4:00 to 9:00, other time off, through the relay to control the light source to achieve the switch state.

Power supply module

In the system design mainly by the SCM, sensors, relays three parts, these parts of the power requirements are not the same. The sensor, SCM work in the $5 \mathrm{vDV}$, the relay needs + -12 VDC power supply, power system is 220V AC, Can not meet the needs of the system, so to select the new appropriate power supply.

By the linear DC power supply and switching power supply two categories. Linear power is the AC voltage in the lower voltage range after the rectifier circuit to get the pulse DC voltage, which can achieve a higher degree of stability, but the transformer bulky, the input voltage requirements of a higher range. Switching power supply through the circuit control transistor breaking, high frequency band, small size, high efficiency, from the main circuit, control circuit, detection circuit and auxiliary power of several parts, the design selection Q-100D switching power supply.

Implementation of communication

The computer can send control information to the coordinator in the network through the serial interface, and the coordinator sends the data collected by the network to the computer through the serial port. Data serial communication uses a data line, the data transmission bit 
by bit, each bit of data transmission occupy a fixed period of time. The exchange of information between the system required less data lines for computers, computers and peripherals between the long-distance communication. Serial communication not only supports long distance transmission, high reliability, and cost savings should be extensive. Serial interface standard to select RS-485, it is based on the RS-232 to increase the multi-point, two-way communication features, RS-485 transceiver common two lines so can not send and receive at the same time, using shielded twisted pair transmission.

\section{Summary}

In this paper, high-precision light sensor, to avoid the D / A conversion error, improve the light intensity measurement range and resolution. With ATMEGA16A-PU as the control center of the data acquisition system, based on CC2530 Zigbee-WIFI wireless network to complete the data acquisition and wireless transmission, the collected lighting values for lighting control hardware design.

\section{References}

[1] Wang Xiaodong; ZigBee-based LED intelligent lighting control system [J]; household appliances; 2014

[2] http://blog.sina.com.cn/s/blog_6eedace40102e1kf.html

[3] Chen Shu-cheng; Yang Zhiyong; Wang Jianjia; Design of Greenhouse Greenhouse Data Acquisition System Based on MSP430 and CC2530 [J]; Electronic Design Engineering; 2014

[4] Yang Yang; intelligent home system application research [J]; electronic test; 2016 years 14 